\title{
Comportement alimentaire du lama en garrigue
}

\author{
S de Rouville, J Goussopoulos
}

INRA-ENSAM, place Viala, 34060 Montpellier cedex, France

Des lamas adultes (7 à 12), à l'entretien, ont été maintenus en permanence durant 2 ans sur une garrigue à chêne kermès aux environs de Montpellier. Ils ont été conduits en pâturage tournant sur 4 parcelles de $7000 \mathrm{~m}^{2}$ en moyenne. Le séjour dans chacune d'entre elles est de 2 mois. Ils ont été pesés tous les mois. Observés du lever au coucher du soleil, 2 fois par mois depuis un mirador, leur activité d'ingestion et leur choix alimentaire (végétation herbacée ou arbustive) ont été enregistrés toutes les 5 min et analysés par heure.

La durée d'ingestion (DI) au cours du temps journalier d'observation varie entre $8 \mathrm{~h}$ à l'entrée et $12 \mathrm{~h}$ à la sortie de la parcelle pour l'ensemble des animaux. Cette variation est liée à la saison, aux disponibilités fourragères et aux individus (de Rouville et al, 1991).

La figure 1 porte sur les comportements de 2 lamas au printemps. Elle met en évidence l'existence de phases soutenues d'activité d'ingestion. À l'entrée dans la parcelle (9/3/90), tous les animaux consomment presque exclusivement la strate herbacée qui est abondante. La DI des herbacées est comprise entre 72 et $90 \%$ pour l'ensemble des animaux quelle que soit la saison. Lorsque la strate herbacée devient peu abondante, en fin de parcelle (11/5/90), le temps passé à ingérer des ligneux est plus important, il est compris entre 18 et $40 \%$ de la DI quelle que soit la saison.

Deux catégories d'animaux se différencient : les "pâtureurs" («P») qui passent plus de $70 \%$ de leur DI sur la strate herbacée (lama 1) et les “débroussaileurs» ( $D$ D) dont la DI se partage plus équitablement entre les herbacées et les ligneux (lama 2) (fig 1). Dans les 2 cas, la DI de ligneux est supérieure à celle des herbacées au début de la journée en fin de parcelle. Ce comportement se poursuit en fin de journée pour le lama 2. Certains animaux ont modifié leur comportement en 2 ans, devenant plus “D”.

Durant une période de faibles ressources (étéautomne 1992), les “P» (4 animaux) ont subi une perte moyenne de poids vif de $15 \%$ et les " $D$ " (5 animaux) une perte de $7 \%$. Des mesures complémentaires d'état corporel (notes variant de 0 à
5) confirment la différence entre les 2 types d'animaux : les "P" sont passés de 1,5 à 1 , les "D" de 2,5 à 2 .

En conclusion, 2 types de comportement chez les lamas ont été mis en évidence : "pâtureur" et "débroussailleur". Des modifications du comportement ont été observées dans le temps, des lamas "P» devenant «D". Le lama aurait un comportement alimentaire plastique et pourrait s'adapter à des milieux végétaux hétérogènes.

De Rouville S, Lapeyronie P, Goussopoulos J (1991) Rythmes d'activités et choix alimentaires du lama en garrigue languedocienne. $4^{\ominus}$ Cong int «Terres de Parcours", Montpellier, 22-26 avril 1991, abstr 273
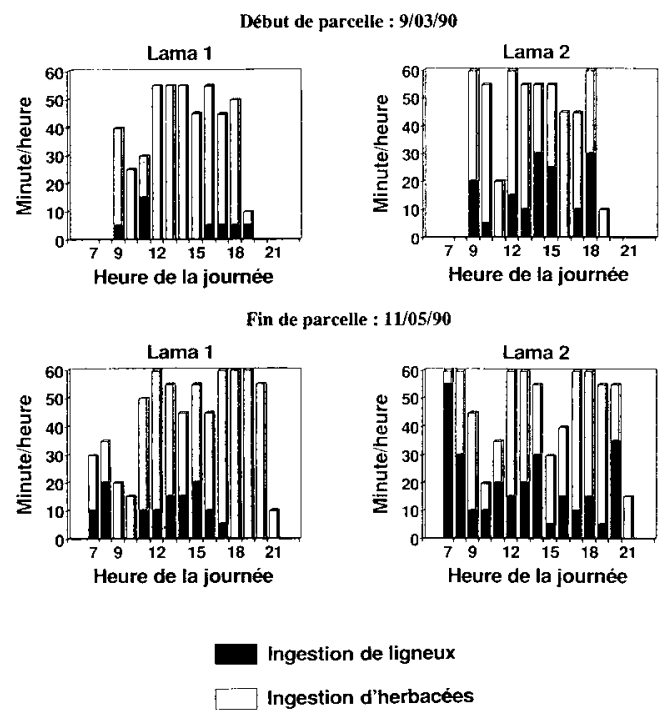

Fig 1. Activité d'ingestion d'un lama "pâtureur» (Lama 1) et d'un lama "débroussailleur" (Lama 2) en début et en fin de parcelle. 